\title{
A Multiplicative Attack Using LLL Algorithm on RSA Signatures with Redundancy
}

\author{
Jean-François Misarsky \\ France Télécom - Branche Développement \\ Centre National d'Etudes des Télécommunications \\ 42, rue des Coutures, B.P. 6243 \\ 14066 Caen Cedex, FRANCE \\ jeanfrancois.misarsky@cnet.francetelecom.fr
}

\begin{abstract}
We show that some RSA signature schemes using fixed or modular redundancy and dispersion of redundancy bits are insecure. Our attack is based on the multiplicative property of RSA signature function and extends old results of De Jonge and Chaum [DJC] as well as recent results of Girault and Misarsky [GM]. Our method uses the lattice basis reduction [LLL] and algorithms of László Babai [B]. Our attack is valid when the length of redundancy is roughly less than half the length of the public modulus. We successfully apply our attack to a scheme proposed for discussion inside ISO. Afterwards, we also describe possible adaptations of our method to attack schemes using mask or different modular redundancies. We explain limits of our attack and how to defeat it.
\end{abstract}

Keywords. Multiplicative attack, LLL algorithm, redundancy, RSA.

\section{Introduction}

Let $n$ be a RSA modulus [RSA], $e$ the public exponent, and $d$ the secret exponent. We can define $P(x)=x^{e}(\bmod n)$ the public function and $S(x)=x^{d}(\bmod n)$ the secret one. The multiplicative property of RSA, i.e. the fact that $S(x y)=S(x) S(y)(\bmod n)$, leads to potential weaknesses, especially when used for signatures. We will make an extensive use of this property in our attack.

When a forger wants the signature of a message $m$, he generates two messages $x$ and $y$ that satisfy $m=x y(\bmod n)$. If he obtains the signatures of $x$ and $y$, as exponentiation preserves the multiplicative structure of the input, he simply computes the signature of $m$ as the product of $S(x)$ and $S(y), S(m)=S(x) S(y)(\bmod n)$. This is a chosen-message attack.

Two standard ways exist to eliminate this potential weakness. One is to sign a hashed value of the message rather than the message itself. The other is to add some redundancy to the message to be signed. These different signature schemes are sometimes called, respectively, schemes with appendix and schemes with message recovery ([MOV], pp.428-432). 
Only the redundancy solution is concerned by this paper. It is of particular interest when the message is short, because it prevents from specifying and implementing a hash-function (a rather delicate cryptographic challenge), and it allows to construct very compact signed messages, since messages can be recovered from the signatures themselves (and hence need not any longer be transmitted or stored). Let $R$ be the invertible redundancy function. The signature of a message $m$ is $\Sigma(m)=S[R(m)]$ and the signer only sends $\Sigma(m)$ of the receiver. The latter applies $P$ to $\Sigma(m)$, and verifies that the result complies with the redundancy rule, i.e. is an element of the image set of $R$. Then he recovers $m$ by discarding the redundancy, i.e. by applying $R^{-1}$ to this result. At Crypto' 85 conference, De Jonge and Chaum [DJC] showed that simple redundancy does not avoid all the chosen-message attacks. In their paper, they show that it is not sufficient to append trailing ' 0 ' bits to the right or the left of the message. They study the case when redundancy is an affine function of $m$, i.e. the signature $\Sigma(m)$ to $m$ is computed as $\Sigma(m)=S(\omega m+a)$. Their attack is based on Euclid's algorithm and is valid for any message $m$ for:

- $a=0$, and any value of $\omega$ such that the amount of redundancy is less than half the length of the public modulus $n$.

- $\omega=1$, a small value of $a$, and when the amount of redundancy is less than one third of the length of the public modulus $n$.

Girault and Misarsky [GM] recently extended these results. Their attack uses an affine variant of Euclid's algorithm due to Okamoto and Shiraishi [OS]. It is valid for any constant $\omega$, any constant $a$, any message $m$ provided that the amount of redundancy is less than half the length of the public modulus $n$. Moreover, they study the case when modular redundancy is used, i.e. when the amount of redundancy is obtained by appending to $m$ the remainder of $m$ modulo some fixed value. In this case, the signature is still subject to a chosen-message attack when redundancy is less than half the length of the public modulus, minus the length of remainder. They give three solutions that prevent their attack; one of them consists in dispersing the message in different parts and another one in using two different modular redundancies.

We show in this paper that a multiplicative attack is feasible on signature scheme that uses dispersion of redundancy bits and fixed or modular redundancy. We precisely explain our attack in this case. But our attack is also valid on more simple schemes or schemes with mask or different modular redundancies.

Our method makes use of the lattice basis reduction, which has not been used in multiplicative attacks yet. But, lattice reduction has already been applied successfully in cryptoanalysis: against Merkle-Hellman public key cryptosystem [S], against Okamoto's cryptosystems [VGT1], against RSA cryptosystem with small exponent $[\mathrm{H}]$, or against RSA encryption with small exponents and random padding $[\mathrm{C}]$, for instance.

We successfully apply our method on ISO 9796 Part 3, Working Draft, December 1996 [ISO2], a scheme using dispersion of redundancy bits and modular redundancy. Afterwards, we explain limits of our attack and how to defeat it.

Throughout this paper, we call bitlength (or length in short) of an integer the number of bits of its binary representation. We denote by $|m|$ the bitlength of $m$. 


\section{Our Results}

We describe a method using lattice basis reduction that finds solutions $x$ and $y$ of the equation $R(m) R(x)=R(y)(\bmod n)$ where:

- $R$ is a redundancy function

- $m$ is a message of which we want to forge a signature

If signatures of $x$ and $y$ can be obtained, i.e. respectively $\Sigma(x)=S(R(x))(\bmod n)$ and $\Sigma(y)=S(R(y))(\bmod n)$, then the signature of $m$ can be easily forged:

In the sequel, we denote by:

$$
\Sigma(m)=\frac{\Sigma(y)}{\Sigma(x)}(\bmod n)
$$

$\omega_{1}, \omega_{2}, \ldots$ : miscellaneous multiplicative redundancies constants

a : fixed redundancy constant

$m \quad:$ a message

$k_{1} \quad:$ the number of parts of $m$

$m_{i} \quad:$ the $i^{\text {th }}$ part of $m$. The message $m$ is split up into $k_{1}$ parts which have not necessary the same length:
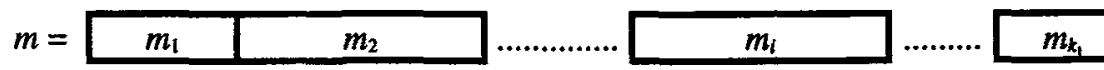

$\varphi(m) \quad:$ modular redundancy of the message $m$ i.e. the remainder of $m$ modulo a fixed value

$k_{2} \quad:$ the number of parts of $\varphi(m)$

$\varphi(m)_{j} \quad:$ the $j^{\text {th }}$ part of $\varphi(m)$. The modular redundancy is split up into $k_{2}$ parts which have not necessary the same length:

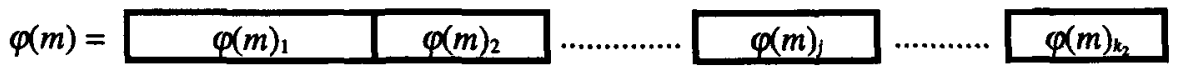

$\begin{array}{ll}n & : \text { RSA modulus } \\ m_{r} & : \text { redundancy modulus }\left(\varphi(m)=m\left(\bmod m_{r}\right)\right)\end{array}$

The redundancy function $R$ can take several forms, with increasing complexity:

i) $\quad R(m)=\omega m+a$

ii) $\quad R(m)=\omega_{1} m+\omega_{2} \varphi(m)+a$

iii) $R(m)=\sum_{i=1}^{k_{1}} m_{i} \omega_{i}+a$

iv) $R(m)=\sum_{i=1}^{k_{1}} m_{i} \omega_{i}+\sum_{j=1}^{k_{2}} \varphi(m)_{j} \omega_{j+k_{1}}+a$

The case iv) generalizes the others and we only study it in the sequel.

Example: when all $\omega_{1}$ are powers of two in the case iv), one could have:

\begin{tabular}{|c|c|c|c|c|c|c|c|c|c|}
\hline$R(m)=$ & $10111 \ldots$ & $m_{1}$ & $\varphi(m)_{1}$ & $. .1001 .$. & $\varphi(m)_{i}$ & $m_{1}$ & $\varphi(m)_{i+1}$ & $\ldots$ & ..1011.. \\
\hline with $a$ & $10111 \ldots$ & $0 . .0$ & $0 \ldots \ldots 0$ &. .1001. & $0 . \ldots \ldots 0$ & $0 . .0$ & $0 \ldots \ldots . . .0$ & $\ldots$ & $. .1011 .$. \\
\hline
\end{tabular}


Remark: we call the number of bits of redundancy the length of $n$ minus the length of $m$. Note that the number of bits of modular redundancy is included in the number of bits of redundancy.

\section{Main result:}

If a signature scheme uses this kind of redundancy function:

$$
R(m)=\sum_{i=1}^{k_{1}} m_{i} \omega_{1}+\sum_{j=1}^{k_{2}} \varphi(m)_{j} \omega_{j+k_{1}}+a
$$

then our attack is valid when the number of bits of redundancy is roughly less than half the length of the public modulus $n$, minus the number of bits of modular redundancy (when the latter is present):

$$
\mid \text { redundancy }\left|<\frac{1}{2}\right| n|-| m_{r} \mid
$$

Another version of our attack, requiring more computation and memory, is valid when the number of bits of redundancy is roughly less than half the length of the public modulus $n$.

\section{System: Definition and Solution}

Solving $R(m) R(x)=R(y)(\bmod n)$ is equivalent to finding the different parts of $R(x)$ and $R(y)$, i.e. respectively $\left(x_{i}\right)_{1} s_{i} k_{1},\left(\varphi(x)_{j}\right)_{1 s_{j} s_{2}}$, and $\left(y_{i}\right)_{1 \leq i} s_{k_{1}},\left(\varphi(y)_{j}\right)_{1} \leqslant_{j} \leqslant_{k_{2}}$.

Let $\left(X_{i}\right)_{1} \leq_{i} s_{k}$ be the different parts of $R(x)$ to find, i.e. all or only part of $\left(x_{i}\right)_{1} s_{i} s_{k_{1}}$ and $\left(\varphi(x)_{j}\right)_{1 \leq j \leq k_{2}}$. Let $\left(Y_{i}\right)_{1 s_{1} s_{k}}$ be the different parts of $R(y)$ to find, i.e. all or only part of $\left(y_{i}\right)_{1 \leq i \leq k_{1}}$ and $\left(\varphi(y)_{j}\right)_{1 \leq j \leq k_{2}}$. The modular redundancy, the fact that $x=\varphi(x)\left(\bmod m_{r}\right)$ and $y=\varphi(y)\left(\bmod m_{r}\right)$, implies two equations:

$$
\begin{aligned}
a_{1} X_{1}+a_{2} X_{2}+\ldots+a_{k-1} X_{k-1} & =X_{k}+h_{1}\left(\bmod m_{r}\right) \\
b_{1} Y_{1}+b_{2} Y_{2}+\ldots+b_{k-1} Y_{k-1} & =Y_{k}+h_{2}\left(\bmod m_{r}\right)
\end{aligned}
$$

with $\left(a_{i}\right)_{1 \leq i \leq k-1},\left(b_{i}\right)_{1 \leq i \leq k-1}, h_{1}$ and $h_{2}$ fixed integers.

\section{Note that:}

- $h_{1}$ and $h_{2}$ are present only when some parts of $R(x)$ and $R(y)$ are fixed, i.e. one or several $x_{i}, \varphi(x)_{j}, y_{i}$ or $\varphi(y)_{j}$ are fixed.

- our method requires the coefficients of $X_{k}$ and $Y_{k}$ to be equal to one. It is easily obtained by a division modulo $m_{r}$. We have deliberately omitted to describe this step.

$R(m) R(x)=R(y)(\bmod n)$ also implies an other equation:

$$
c_{1} X_{1}+c_{2} X_{2}+\ldots+c_{k} X_{k}+d_{1} Y_{1}+d_{2} Y_{2}+\ldots+d_{k-1} Y_{k-1}=Y_{k}+h_{3}(\bmod n)
$$

with $\left(c_{i}\right)_{1 \leq i \leq k},\left(d_{i}\right)_{1 \leq i \leq k-1}$ and $h_{3}$ fixed integers. 
Let $(S I)$ be the system:

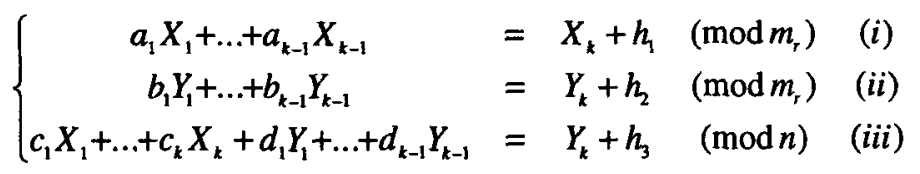

$(S I)$ is a system with constraints on value of $\left(X_{i}\right)_{1} s_{i} s_{k}$ and $\left(Y_{i}\right)_{1} s_{i} s_{k}$.

We have for $1 \leq i \leq k$ :

$$
\begin{aligned}
& 0 \leq X_{i}<2^{\text {Lenght of the part } X_{i} \text { in bust }} \\
& 0 \leq Y_{i}<2^{\text {Lenght of the part } Y_{i} \text { in bis }}
\end{aligned}
$$

When modular redundancy is not used in the signature scheme, $(i)$ and (ii) are useless. Only (iii) is necessary.

In the first part of our study, we define a lattice where all points give a solution to this system without second member, $h_{1}=h_{2}=h_{3}=0$, and without constraints on values of

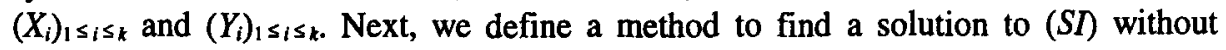
constraints on values of $\left(X_{i}\right)_{1} s_{i} s_{k}$ and $\left(Y_{i}\right)_{1} s_{i} s_{k}$ by using this lattice. After, we explain how to obtain solutions to the system $(S I)$ with additional constraints on values of $\left(X_{i}\right)_{1} s_{i}$ and $\left(Y_{i}\right)_{1} s_{i}$. Finally we study the efficiency of our method.

\subsection{First Step: Determination of the Lattice}

We define an integer lattice $L$ such that any element of this lattice is solution to $(S)$.

$(S)$ is the system $(S I)$ without second member and without constraints on values of $\left(X_{i}\right)_{1} s_{i} s_{k}$ and $\left(Y_{i}\right)_{1} s_{i} s_{k}$.

$$
\left\{\begin{array}{cccc}
a_{1} X_{1}+\ldots+a_{k-1} X_{k-1} & = & X_{k}\left(\bmod m_{r}\right) \\
b_{1} Y_{1}+\ldots+b_{k-1} Y_{k-1} & = & Y_{k}\left(\bmod m_{r}\right) \\
c_{1} X_{1}+\ldots+c_{k} X_{k}+d_{1} Y_{1}+\ldots+d_{k-1} Y_{k-1} & = & Y_{k} & (\bmod n)
\end{array}\right.
$$

Hence, we define a lattice $L$ of dimension $2 k$ such that any vector $v=\left(v_{1}, v_{2}, \ldots, v_{2 k-1}\right.$, $v_{2 x}$ ) verifies:

$$
\begin{aligned}
a_{1} v_{1}+a_{2} v_{2}+\ldots+a_{k-1} v_{k-1} & =v_{k}\left(\bmod m_{r}\right) \\
b_{1} v_{k+1}+b_{2} v_{k+2}+\ldots+b_{k-1} v_{2 k-1} & =v_{2 k}\left(\bmod m_{r}\right) \\
c_{1} v_{1}+c_{2} v_{2}+\ldots+c_{k} v_{k}+d_{1} v_{k+1}+d_{2} v_{k+2}+\ldots+d_{k-1} v_{2 k-1} & =v_{2 k}(\bmod n)
\end{aligned}
$$

Let $M$ be the matrix of lattice $L$. Columns vectors of $M$ are a basis of $L$, and for any element $v$ of $L$, there is a column vector $\alpha$ with integer components such that:

$$
M \alpha=v
$$

Now, we construct this matrix $M$.

We denote by $M_{1}$ an identity matrix of dimension $2 k$ where the row $k$ is replaced by:

$$
\left(a_{1}, a_{2}, \ldots, a_{k-1}, m_{m}, 0,0, \ldots, 0\right)
$$

Then, for any vector $\alpha$ with integer components, $v=M_{1} \alpha$ is a vector with components satisfying (a). Remark that $v_{t}=\alpha_{i}$ with $1 \leq i \leq 2 k, i \neq k$ and: 


$$
v_{k}=a_{1} v_{1}+a_{2} v_{2}+\ldots+a_{k-1} v_{k-1}+\alpha_{k} m_{r}
$$

We gather equations $(b)$ and $(c)$ together with the Chinese Remainder Theorem. It is possible because $n$ is the public modulus of RSA and is prime with $m_{r}$ (otherwise we have a factor of $n$ !).

We denote by Chinese the Chinese remainder function:

Chinese $(a(\bmod m), b(\bmod n))$, with $m$ and $n$ relatively primes, returns $x$ such that:

$$
\left\{\begin{array}{l}
x=a(\bmod m) \\
x=b(\bmod n)
\end{array}\right.
$$

Let $\left(f_{i}\right)_{1} \leq 1 \leq 2 k$ such that:

We obtain:

$$
\begin{array}{ll}
f_{i}=\text { Chinese }\left(0\left(\bmod m_{r}\right), c_{i}(\bmod n)\right) & \text { when } 1 \leq i \leq k \\
f_{i}=\operatorname{Chinese}\left(b_{i-k}\left(\bmod m_{r}\right), d_{i k}(\bmod n)\right) & \text { when } k+1 \leq i \leq 2 k-1
\end{array}
$$

$$
f_{1} v_{1}+f_{2} v_{2}+\ldots+f_{k-1} v_{k-1}+f_{k} v_{k}+f_{k+1} v_{k+1}+f_{k+2} v_{k+2}+\ldots+f_{2 k-1} v_{2 k-1}=v_{2 k}\left(\bmod m_{r} n\right)
$$

But $\alpha_{k}$ is different from $v_{k}$. We use (d) to replace $\nu_{k}$ in $(f)$. We finally obtain an equation (e), equivalent to $(b)$ and $(c)$, that has this form:

$$
e_{1} v_{1}+e_{2} v_{2}+\ldots+e_{k-1} v_{k-1}+e_{k} \alpha_{k}+e_{k+1} v_{k+1}+e_{k+2} v_{k+2}+\ldots+e_{2 k-1} v_{2 k-1}=v_{2 k}\left(\bmod m_{r} n\right)
$$

with:

$$
\begin{array}{ll}
e_{i}=f_{i}+a_{i} f_{k} & \text { when } 1 \leq i \leq k-1 \\
e_{k}=f_{k} m_{r} & \text { when } k+1 \leq i \leq 2 k-1 \\
e_{i}=f_{i} &
\end{array}
$$

Finally, the matrix $M$ is the matrix $M_{1}$ where the latest row is replaced by the vector:

We have:

$$
\left(e_{1}, e_{2}, \ldots, e_{k-1}, e_{k}, e_{k+1}, \ldots, e_{2 k-1}, m_{r} n\right)
$$

$$
M=\left(\begin{array}{ccccccccc}
1 & 0 & \ldots & \ldots & \ldots & \ldots & \ldots & \ldots & 0 \\
0 & \ddots & \ddots & & & & & & \vdots \\
\vdots & \ddots & \ddots & \ddots & & & & & \vdots \\
0 & \ldots & 0 & 1 & \ddots & & & & \vdots \\
a_{1} & \ldots & \ldots & a_{k-1} & m_{r} & 0 & & & \vdots \\
0 & \ldots & \ldots & \ldots & 0 & 1 & \ddots & & \vdots \\
\vdots & & & & & \ddots & \ddots & 0 & \vdots \\
0 & \ldots & \ldots & \ldots & \ldots & \ldots & 0 & 1 & 0 \\
e_{1} & e_{2} & \ldots & \ldots & \ldots & \ldots & e_{2 k-2} & e_{2 k-1} & m_{r} n
\end{array}\right)
$$

A solution to the system (S) is obtained by multiplying matrix $M$ by an integer vector $\alpha$. The result $v$ gives a solution to $(S): v_{1}, \ldots, v_{k}$ will be $X_{1}, \ldots, X_{k}$ and $v_{k+1}, \ldots, v_{2 k}$ will be $Y_{1}, \ldots ., Y_{k}$. The reciprocal can be easily demonstrated and consequently we have: 


\section{Proposition 3.1.1:}

$A$ vector is in $L$ if and only if it is a solution to $(S)$.

\subsection{Second step: System with a Second Member}

Let $\left(S^{\prime}\right)$ be the system $(S)$ with a second member. $\left(S^{\prime}\right)$ is the initial system $(S I)$ but without constraints on values of solutions. The same lattice $L$ is used to solve $\left(S^{\prime}\right)$.

\section{Proposition 3.2.1:}

$$
\begin{aligned}
& \text { Let } v=\left(v_{1}, v_{2}, \ldots, v_{2 k-l}, v_{2 k}\right) \text { be a vector of } L \\
& \text { Let } P=\left(0,0, \ldots, 0, p_{k}, 0, \ldots, 0, p_{2 k}\right) \text { with } \\
& \qquad \begin{array}{l}
p_{k} \\
p_{2 k}
\end{array}=h_{1}
\end{aligned}
$$

Then $\beta=v-P$ gives a solution to ( $\left.S^{\prime}\right)$.

$\beta_{1}, \ldots, \beta_{k}$ will be $X_{l}, \ldots, X_{k}$ and $\beta_{k+1}, \ldots, \beta_{2 k}$ will be $Y_{1}, \ldots, Y_{k}$

\section{Proof:}

$$
\begin{aligned}
\beta_{i} & =v_{i} \quad \text { when } i \in\{1,2,3, \ldots, k-1, k+1, k+2, \ldots, 2 k-1\} \\
\beta_{k} & =v_{k}-p_{k} \\
& =a_{1} v_{1}+a_{2} v_{2}+\ldots+a_{k-1} v_{k-1}+\alpha_{k} m_{r}-h_{1} \\
& =a_{1} \beta_{1}+a_{2} \beta_{2}+\ldots+a_{k-1} \beta_{k-1}+\alpha_{k} m_{r}-h_{1} \\
\beta_{2 k} & =v_{2 k}-p_{2 k} \\
& =e_{1} v_{1}+\ldots+e_{k-1} v_{k-1}+e_{k} c_{k}+e_{k+1} v_{k+1}+e_{k+2} v_{k+2}+\ldots+e_{2 k-1} v_{2 k-1}+\alpha_{2 k} m_{r} n-p_{2 k}
\end{aligned}
$$

And we have:

$$
\begin{aligned}
\beta_{2 k}\left(\bmod m_{r}\right) & =b_{1} v_{k+1}+b_{2} v_{k+2}+\ldots+b_{k-1} v_{2 k-1}-h_{2} \\
& =b_{1} \beta_{k+1}+b_{2} \beta_{k+2}+\ldots+b_{k-1} \beta_{2 k-1}-h_{2} \\
\beta_{2 k}(\bmod n) & =c_{1} v_{1}+c_{2} v_{2}+\ldots+c_{k} v_{k}+d_{1} v_{k+1}+\ldots+d_{k-1} v_{2 k-1}-h_{3}-c_{k} h_{3} \\
& =c_{1} \beta_{1}+c_{2} \beta_{2}+\ldots+c_{k}\left(v_{k}-h_{1}\right)+d_{1} \beta_{k+1}+\ldots+d_{k-1} \beta_{2 k-1}-h_{3}
\end{aligned}
$$

As $v_{k}-h_{1}=v_{k}-p_{k}=\beta_{k}$, we have:

$\beta_{2 k}(\bmod n)=c_{1} \beta_{1}+c_{2} \beta_{2}+\ldots+c_{k} \beta_{k}+d_{1} \beta_{k+1}+\ldots+d_{k-1} \beta_{2 k-1}-h_{3}$

Thus, $\beta$ gives a solution to $\left(S^{\prime}\right)$.

\subsection{Third step: Additional Constraints}

We always consider the system $\left(S^{\prime}\right)$, but we take into account the initial constraints on values of $\left(X_{i}\right)_{1 \leq_{i} \leq_{k}}$ and $\left(Y_{i}\right)_{1} \leq_{i} s_{k}$. Hence, we solve $(S I)$.

\section{First case: same bounds}

Let $B$ be a positive integer. Find $X_{i}$ and $Y_{i}$ such that $0 \leq X_{i} \leq B$ and $0 \leq Y_{i} \leq B$ for any $i$ such that $1 \leq i \leq k$.

\section{Proposition 3.3.1:}

Let $H C$ be a ball of radius $B / 2$, relative to the norm sup, centred on $Q=P+(B / 2$, $B / 2, \ldots, B / 2$ ), where the point $P$ is defined in the proposition 3.2.1. Let $v$ be a vector of $L$ inside $H C$, and $\beta=v-P$.

Then $\beta$ gives a solution to ( $\left.S^{\prime}\right)$ and satisfies additional constraints. 


\section{Proof:}

Proposition 3.2.1 shows that $\beta$ gives a solution to $\left(S^{\prime}\right)$.

$v$ inside $H C$ implies $0 \leq v_{i}-p_{t} \leq B$, i.e. $0 \leq \beta_{i} \leq B$, for any $1 \leq i \leq 2 k$.

\section{Second case: distinct bounds}

Let $\left(B_{i}\right)_{1} \leq_{i} \leq_{2 k}$ be a family of positive integers. Find $X_{i}$ and $Y_{i}$ such that: $0 \leq X_{i} \leq B_{i}$ and $0 \leq Y_{i} \leq B_{k+i}$ for any $i$ such that $1 \leq i \leq k$.

We apply a method of expansion-contraction to the lattice $L$ to obtain another lattice $L^{\prime}$, see [VGT1] and [VGT2] for more details. We denote by $M^{\prime}$ the matrix of lattice $L^{\prime}$.

Define $B$ as $B^{2 k}=\prod_{i=1}^{2 k} B_{i}$. Let $\left(\lambda_{i}\right)_{1} s_{i} s_{2 k}$ such that $\lambda_{i}=\frac{B}{B_{i}}$. Then the product $\prod_{i=1}^{2 k} \lambda_{i}$ is equal to $1 . M^{\prime}$ is the matrix $M$ where each row $i, 1 \leq i \leq 2 k$, is multiplied by $\lambda_{i}$ :

$$
M^{\prime}=\left(\begin{array}{ccccccccc}
\lambda_{1} & 0 & \ldots & \ldots & \ldots & \ldots & \ldots & \ldots & 0 \\
0 & \ddots & \ddots & & & & & & \vdots \\
\vdots & \ddots & \ddots & \ddots & & & & & \vdots \\
0 & \ldots & 0 & \lambda_{k-1} & 0 & & & & \vdots \\
\lambda_{k} a_{1} & \ldots & \ldots & \lambda_{k} a_{k-1} & \lambda_{k} m_{r} & 0 & & & \vdots \\
0 & \ldots & \ldots & \ldots & 0 & \lambda_{k+1} & 0 & & \vdots \\
\vdots & & & & & \ddots & \ddots & \ddots & \vdots \\
0 & \ldots & \ldots & \ldots & \ldots & \ldots & 0 & \lambda_{2 k-1} & 0 \\
\lambda_{2 k} e_{1} & \lambda_{2 k} e_{2} & \ldots & \ldots & \ldots & \ldots & \lambda_{2 k} e_{2 k-2} & \lambda_{2 k} e_{2 k-1} & \lambda_{2 k} m_{r} n
\end{array}\right)
$$

Remark that $\operatorname{det}(M)=\operatorname{det}\left(M^{\prime}\right)=m_{r}^{2} n$.

\section{Proposition 3.3.2:}

Let $P^{\prime}=\left(0,0, \ldots, 0, p_{k} \lambda_{k}, 0, \ldots, 0, p_{2 k} \lambda_{2 k}\right)$, where $P$ is defined in the proposition 3.2.1. Let $H C$ be a ball, relative to the norm sup, of radius $B / 2$ centred on $Q=P^{\prime}+(B / 2, B / 2, \ldots, B / 2)$. Let $v^{\prime}$ be an element of $L^{\prime}$ inside $H C$ and $\beta^{\prime}=v^{\prime}-P^{\prime}$. Then $\beta=\left(\lambda_{1}^{-1} \beta_{1}^{\prime}, \ldots, \lambda_{2 k}^{-1} \beta_{2 k}^{\prime}\right)$ gives a solution to $\left(S^{\prime}\right)$ and satisfies additional constraints.

\section{Proof:}

$$
\begin{aligned}
\beta=\left(\lambda_{1}^{-1} \beta_{1}, \ldots, \lambda_{2 k^{-1}} \beta_{2 k^{\prime}}\right) & =\left(\lambda_{1}^{-1}\left(v_{1}^{\prime}-p_{1}^{\prime}\right), \ldots, \lambda_{2 k^{-1}}\left(v_{2 k^{\prime}}-p_{2 k^{\prime}}\right)\right) \\
& =\left(\lambda_{1}^{-1} v_{1}^{\prime}-p_{1}, \ldots, \lambda_{2 k^{-1}} v_{2 k}-p_{2 k}\right)
\end{aligned}
$$

Let $v=\left(\lambda_{1}{ }^{-1} v_{1}^{\prime}, \ldots, \lambda_{2 k^{-1}} v_{2 k}{ }^{\prime}\right)$ and $\beta=v-P$.

Then $v \in L$ and proposition 3.2.1 shows that $\beta$ gives a solution to $\left(S^{\prime}\right)$.

$v^{\prime}$ inside $H C$ implies $0 \leq v_{i}^{\prime}-p_{i}^{\prime} \leq B$, i.e. $0 \leq \beta_{i} \leq B_{i}$, for any $1 \leq i \leq 2 k$. 
Remark: the first case is a particular case of the second. In the sequel, we will consider always the lattice $L^{\prime}$ and its matrix $M^{\prime}$.

\subsection{How to Generate a Solution?}

Proposition 3.3.2 shows that a point of lattice $L$ ' inside $H C$ gives a solution to the system (SI). To find one, take a point $x$ inside $H C$ and find a close lattice point inside HC.

First, apply the LLL algorithm [LLL] to the matrix $M^{\prime}$. A reduced basis of $L^{\prime}$ is obtained. Next, apply one of two algorithms of László Babai, Rounding Off or Nearest Plane, described in [B] to find a solution.

Let $u$ be the nearest lattice point of $x$ and $d$ the dimension of lattice $L$ '.

- ROUNDING OFF: this algorithm finds a lattice point $v$ ' such that:

$$
\left|x-v^{\prime}\right| \leq \mathrm{C}_{d}|x-u| \text { with } \mathrm{C}_{d}=1+2 d(9 / 2)^{d / 2}
$$

- NEAREST PLANE: this algorithm finds a lattice point $v^{\prime}$ such that:

$$
\left|x-v^{\prime}\right| \leq \mathrm{C}_{d}|x-u| \text { with } \mathrm{C}_{d}=2^{d / 2}
$$

Remark that, if the dimension $d$ of lattice increases, then the probability that one of these algorithms finds a lattice point inside $H C$ decreases.

\subsection{Efficiency: Heuristic Approach}

Heuristically, if the ratio of the $H C$ volume to the lattice determinant is greater than 1 , then there is at least one lattice point in $H C$.

The Nearest Plane algorithm certainly finds this point when the dimension $d$ of the lattice is not too large. When $d$ increases, the term $\mathrm{C}_{d}=2^{d / 2}$ increases too, and the probability to obtain a point inside $H C$ decreases.

We study the general case where the redundant version of $m$ is:

$$
R(m)=\sum_{i=1}^{k_{1}} m_{i} \omega_{i}+\sum_{j=1}^{k_{2}} \varphi(m)_{j} \omega_{j+k_{i}}+a
$$

This is the most complicated case, and the solutions to the others can be derived from the following analysis.

We denote by:

$$
\begin{aligned}
& t: \text { the bitlength of } n \\
& b_{i}: \text { the length of the part } m_{i} \text { of } m \\
& b: \text { such that } \sum_{l=1}^{k_{1}} b_{i}=b \\
& c_{j}: \text { the length of the part } \varphi(m)_{j} \text { of } \varphi(m) \\
& c: \text { such that } \sum_{j=1}^{k_{2}} c_{j}=c
\end{aligned}
$$

\section{First method: modular redundancies are fixed}

Modular redundancies $\varphi(x)$ and $\varphi(y)$ are fixed. Finding two messages $x$ and $y$ such that $R(m) R(x)=R(y)(\bmod n)$ is equivalent to solve $(S I)$ with $k=k_{1}$.

Lattice dimension $\quad: d=2 k_{1}$

Lattice determinant $: \operatorname{det}(L)=\operatorname{det}\left(L^{\prime}\right)=m_{r}{ }^{2} n<\left(2^{c}\right)^{2} 2^{\prime}$ 
$H C$ volume $\quad:\left(2^{\frac{2 \sum_{i}}{d}}\right)^{d}=2^{2 b}$

Heuristically, there is one point in $H C$ if:

$$
\begin{aligned}
\frac{2^{2 b}}{2^{2 c+t}} & >1 \\
2 b & >2 c+t \\
b & >\frac{t}{2}+c \\
t-b & <\frac{t}{2}-c \\
\mid \text { redundancy } \mid & <\frac{1}{2}|n|-\left|m_{r}\right|
\end{aligned}
$$

Second method: modular redundancies are not fixed

Finding two messages $x$ and $y$ such that $R(m) R(x)=R(y)(\bmod n)$ is equivalent to solve ( $S I)$ with $k=k_{1}+k_{2}$. But, there is a disadvantage when modular redundancies are not fixed. The dimension of lattice increases and therefore the probability to find a lattice point in $H C$ with Babai's algorithms decreases.

Lattice dimension $: d=2\left(k_{1}+k_{2}\right)$

Lattice determinant $: \operatorname{det}(L)=m_{r}{ }^{2} n<\left(2^{c}\right)^{2} 2^{\prime}$

$H C$ volume

$$
:\left(2^{2\left(\sum_{i} b_{i}+\sum_{j} c_{j}\right)}\right)^{d}=2^{2(b+c)}
$$

Heuristically, there is one point in $H C$ if:

$$
\begin{aligned}
\frac{2^{2(b+c)}}{2^{2 c+t}} & >1 \\
2(b+c) & >2 c+t \\
b & >\frac{t}{2} \\
t-b & <\frac{t}{2}
\end{aligned}
$$

$\mid$ redundancy $\left|<\frac{1}{2}\right| n \mid$ 


\section{Application}

We applied our attack on a project of digital signature schemes giving message recovery ISO/IEC 9796-3, Working Draft, December 1996 [ISO2]. It is supposed to avoid the known attacks against RSA [GQLS]. This part of ISO/IEC 9796 specifies a digital signature scheme for messages of limited length, so that the message is completely recovered from the signature. It uses a check-function to save bits and computations. This check-function is a modular redundancy, it is the remainder of the message to be signed modulo $2^{79}+1$. The modular redundancy takes the form:

$$
R(m)=\sum_{i=1}^{k_{1}} m_{i} \omega_{i}+\sum_{j=1}^{k_{2}} \varphi(m) \omega_{j+k_{1}}+a
$$

with all $\left(\omega_{i}\right)_{1 \leq i \leq k_{1}+k_{2}}$ powers of two. We experiment our attacks on this scheme with a public modulus $n$ of 640 bits of length. In this case the project defines an intermediate string $I S$ :

Structure of the intermediate string IS $(640-80=560$ bits $)$

\begin{tabular}{|c|c|c|c|}
\hline Header & Padding Field & Data field & Trailer \\
\hline Three bits & $640-k_{m}-87$ bits & $k_{m}$ bits & Four bits \\
\hline Set at 010 & $\begin{array}{c}640-k_{m}-88 \text { bits set to } 0 \text { followed } \\
\text { by one bit set to } 1\end{array}$ & Message $m$ & Set to 0110 \\
\hline
\end{tabular}

The structure of the valid message (640 bits) is:

Binary pattern (check-code in bold)

$$
12+4+28+4+28+4+\ldots+28+4+28+4+16=640 \text { bits }
$$

We applied the first method, i.e. we fixed the check-code. We found several solutions by using the Rounding Off algorithm. We give an example of solution:

Public modulus:

$$
\begin{aligned}
& n=\text { ffffffff } 78 f 6 c 555 \quad 06 c 59785 \text { e871211e } \\
& \text { e120b0b5 dd644aa7 96d82413 a47b2457 } \\
& 3 \text { f1be574 5b5cd995 of } 6 \mathrm{~b} 389 \mathrm{~b} \quad 52350 \mathrm{~d} 4 \mathrm{e} \\
& 01 e 90009 \quad 669 a 8720 \text { bf265a28 } 65994190 \\
& \text { a661dea3 c7828e2e 7ca1b196 5ladc2d5 }
\end{aligned}
$$

Message $m$ :

$$
m=\begin{array}{llll}
\text { fedcba98 } & 76543210 & \text { fedcba98 } & 76543210 \\
\text { fedcba98 } & 76543210 & \text { fedcba98 } & 76543210 \\
\text { fedcba98 } & 76543210 & \text { fedcba98 } & 76543210 \\
\text { fedcba98 } & 76543210 & \text { fedcba98 } & 76543210
\end{array}
$$

Check-code:

$$
c=0 f 6 e \text { af3 a0b1 } 3571358 b
$$


Valid message of $m$ :

\begin{tabular}{|c|c|c|c|c|}
\hline$(m)$ & $\begin{array}{r}=4 \text { bbobbbb } \\
\text { edc } 4 \text { ba } 98 \\
210 \text { afedc } \\
65433210 \\
\text { a9837654 }\end{array}$ & $\begin{array}{l}\text { bbbfafed } \\
765 a 4321 \\
\text { ba908765 } \\
\text { fed5cba9 } \\
32150 \text { fed }\end{array}$ & $\begin{array}{l}\text { cba69876 } \\
\text { Ofefdcba } \\
432 \mathrm{~b} 10 \mathrm{fe} \\
87675432 \\
\text { cba89876 }\end{array}$ & $\begin{array}{l}543 e 210 f \\
98736543 \\
\text { dcb1a987 } \\
10 \mathrm{f} 1 \mathrm{edcb} \\
543 \mathrm{~b} 2106\end{array}$ \\
\hline
\end{tabular}

Message $x$ :

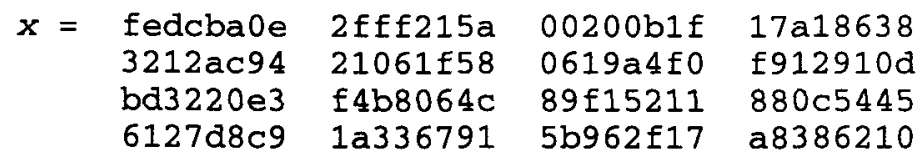

Message $y:$

\begin{tabular}{|c|c|c|c|c|}
\hline$y=$ & $\begin{array}{l}\text { fedcba14 } \\
\text { cd1335d1 } \\
02 \mathrm{~b} 2 \mathrm{f14a} \\
50 \text { f4eaf1 }\end{array}$ & $\begin{array}{l}7597 \mathrm{~b} 137 \\
308 \mathrm{beg} 6 \mathrm{c} \\
39 \mathrm{f} 709 \mathrm{a} 6 \\
1 \mathrm{a} 2 \mathrm{f} 2381\end{array}$ & $\begin{array}{l}39 \mathrm{~d} 20 \mathrm{f} 85 \\
14 \mathrm{~b} 053 \mathrm{~d} 1 \\
\text { e6a0ede5 } \\
064 \mathrm{c} 2 \text { fof }\end{array}$ & $\begin{array}{l}33 \mathrm{~b} 07 \mathrm{f} 20 \\
4230 \mathrm{e} 40 \mathrm{f} \\
\mathrm{ae} 1 \mathrm{f} 6313\end{array}$ \\
\hline
\end{tabular}

Valid message of $x$ :

$\begin{array}{rllll}\operatorname{Sr}(x)= & 4 b \mathrm{~b} 0 \mathrm{bbbb} & \text { bbbfafed } & \text { cba60e2f } & \text { ff2e15a0 } \\ & 02040 \mathrm{~b} 1 \mathrm{f} & 17 \mathrm{aa} 1863 & 832 \mathrm{f} 12 \mathrm{ac} & 94231061 \\ & \text { f58a0619 } & \text { a4f00f91 } & 291 \mathrm{~b} 0 \mathrm{dbd} & 32210 \mathrm{e} f \mathrm{f} \\ & 4 \mathrm{~b} 83064 \mathrm{c} & 89 \mathrm{f5} 1521 & 18870 \mathrm{c} 54 & 4561127 \mathrm{~d} \\ & 8 \mathrm{c} 931 \mathrm{a} 33 & 679515 \mathrm{~b} 9 & 62 \mathrm{f} 817 \mathrm{a} 8 & 386 \mathrm{~b} 2106\end{array}$

Valid message of $y:$

\begin{tabular}{|c|c|c|c|c|}
\hline \multirow[t]{5}{*}{$\operatorname{Sr}(y)$} & $4 \mathrm{bb} 0 \mathrm{bbbb}$ & bbbfafed & cba61475 & $97 \mathrm{~b}$ \\
\hline & 90 & $33 \mathrm{ba} 07 f 2$ & Ocdf1335 & d1 $3308 \mathrm{be}$ \\
\hline & $4 \mathrm{~b}$ & $53 \mathrm{~d} 01423$ & $0 e 4 b 0 f 02$ & $\mathrm{~b} 2 \mathrm{f} 114 \mathrm{a} 3$ \\
\hline & $9 f 7309 a 6$ & e6a50ede & $5 \operatorname{ae} 71 \mathrm{f} 63$ & $13510 \mathrm{f}$ \\
\hline & af $131 \mathrm{a}$ & 23851064 & $22 f 80 f f 3$ & ffab210 \\
\hline
\end{tabular}

We obtained this result within 30 minutes on a Pentium $166 \mathrm{MHz}$ by using GP/PARI CALCULATOR Version 1.39 (ftp: megrez math.u-bordeaux. fr/pub/pari). It is the time necessary to apply LLL algorithm to the initial matrix. After, we can easily obtain different messages $x$ and $y$ in a few seconds by using Rounding Off or Nearest Plane algorithm on different points inside $H C$.

\section{Extensions}

We have described an attack on a signature scheme using one modular redundancy. But it is possible to increase the number of modular redundancies. If the different moduli are relatively prime, they can be gathered into one equation with the Chinese Remainder theorem and solved with the first method. If these moduli are not relatively prime, we use the second method, then the probability to find a solution is lower because the dimension is high. 
We denote by mask a $\boldsymbol{k}_{2}$-bit fixed string. Our attack also succeeds on a scheme that uses a modular redundancy and a mask, i.e. you apply the function exclusive OR between modular redundancy and the mask. In this case we use the first method.

\section{How to defeat this forgery}

If you want to use fixed or modular redundancy, it is recommended to have the same amount of redundancy as the number of bits of message $m$, and to have a big dispersion of redundancy bits. It is not sure that you cannot apply our attack but the probability of success will be small.

Another way to avoid this attack is to split the message and define bits of redundancy as parity bits (such as those determined by Hamming codes) of its different parts. ISO 9796 [ISO1] is another possible solution, but it doubles the length of the bit pattern you sign. Our attack cannot apply to the latter schemes because the redundancy depends on different bits of message $m$ and we cannot adjust our attack to this case.

\section{Conclusion}

This paper describes two attacks to forge a signature of a message $m$ when the bits of redundancy are dispersed and/or when a modular redundancy is used. The first one is valid when the length of redundancy is less than half the length of public modulus, minus the length of modular redundancy. The second attack is valid when the length of redundancy is less than half the length of public modulus, but the probability to find a forgery is smaller (because the lattice dimension grows); however, we have noticed that the Nearest Plane and Rounding Off algorithms [B] generally give better results than expected.

Afterwards, we have briefly described possible adaptations of our method to attack schemes using mask or different modular redundancies. Hence, we have shown the weakness of many attractive redundancy functions for the purpose of RSA digital signatures.

Finally, we advise to use, for RSA signature scheme with fixed or modular redundancy, the same length of redundancy that the length of the message and to disperse message bits in the valid message. But the best solution remains to use ISO 9796 [ISO1] or the parity bits scheme briefly described above, because they apparently cannot be attacked by our techniques.

\section{Acknowledgments}

We would like to thank Marc Girault for encouraging this research and for helpful comments on this paper. We are grateful to Brigitte Vallee for help on the lattice theory and for pointing out corrections to the initial draft. We also thank Louis Guillou for stimulating this research, Jacques Traoré and the referees for their useful comments. 


\section{References}

[B]

[C]

[DJC]

[GQLS]

[GM]

[H]

[ISO1]

[ISO2]

[LLL]

[OS]

[MOV]

[RSA]

[S]

[VGT1]

[VGT2]

L. Babai, "On Lovász' lattice reduction and the nearest lattice point problem", Combinatorica 6, pp. 1-14.

Don Coppersmith, "Finding a Small Root of Univariate Modular Equation", Proceedings of Eurocrypt '96, Lecture Note in Computer Science, vol. 1070, pp. 155-165.

W. De Jonge, D. Chaum, "Attacks on some RSA Signatures", Advances in Cryptology, Crypto '85 Proceedings, Lecture Notes In Computer Science, vol. 218, Springer-Verlag, Berlin, 1986, pp. 18-27.

L.C. Guillou, J.J. Quisquater, P. Landrock, C. Shaer, "Precautions taken against various potential attacks in ISO/IEC DIS 9796, Digital signature scheme giving message recovery", Eurocrypt ' 90 Proceedings, Lecture Notes in Computer Science, vol.473, Springer-Verlag, pp 465-473.

M. Girault, J.F. Misarsky, "Selective Forgery of RSA Signatures Using Redundancy", Advances in Cryptology - Eurocrypt '97, Lecture Notes in Computer Science, vol. 1233, Springer-Verlag, pp 495-507.

J. Hastad, "Solving simultaneous modular equations of low degree", SIAM J. Comput. vol.17, No.2, April 1988.

ISO/IEC 9796, December 1991, "Digital signature scheme giving message recovery".

ISO/IEC 9796-3, Working Draft, December 1996, "Digital signature schemes giving message recovery; Part 3: Mechanisms using a checkfunction".

A. K. Lenstra, H. W. Lenstra, L. Lovász, "Factoring Polynomials with Rational Coefficients", Mathematische Annalen, vol. 261, n. 4, 1982, pp. 515-534.

T. Okamoto and A. Shiraishi, "A fast signature scheme based on quadratic inequalities", Proc. of the 1985 Sympsium on Security and Privacy, Apr.1985, Oakland, CA.

A.J. Menezes, P.C. Van Oorschot, S.A. Vanstone, "Handbook of Applied Cryptography", CRC Press.

R.L. Rivest, A. Shamir and L. Adleman, "A method for obtaining digital signatures and public-key cryptosystems", CACM, Vol. 21, n², Feb. 1978, pp. 120-126.

A. Shamir, "A polynomial-time algorithm for breaking the basic Merkle-Hellman cryptosystem", Proceedings of the $23^{\text {rd }}$ IEEE Symposium on Foundations of Computer Science, pp 145-152. IEEE, 1982.

B. Vallée, M. Girault, P. Toffin, "How to break Okamoto's cryptosystems by reducing lattice bases", Proceedings of Eurocrypt'87, Lecture notes in Computer Science.

B. Vallée, M. Girault, P. Toffin, "How to guess L-th roots modulo $n$ by reducing lattice bases", Proc. of Conference of ISSAC-88 and AAECC6, Jul. 88. 